\title{
Background modelling in very-high-energy $\gamma$-ray astronomy
}

\author{
D. Berge ${ }^{1,2}$, S. Funk ${ }^{1,3}$, and J. Hinton ${ }^{1,4,5}$ \\ 1 Max-Planck-Institut für Kernphysik, PO Box 103980, 69029 Heidelberg, Germany \\ 2 CERN, 1211 Geneva 23, Switzerland \\ e-mail: berge@cern.ch \\ 3 Kavli Institute for Particle Astrophysics and Cosmology, Stanford University, 2575 Sand Hill Road, Menlo Park, CA 94025, USA \\ ${ }^{4}$ Landessternwarte, Königstuhl, 69117 Heidelberg, Germany \\ 5 School of Physics \& Astronomy, University of Leeds, Leeds LS2 9JT, UK
}

Received 31 October 2006 / Accepted 14 February 2007

\begin{abstract}
Context. Ground based Cherenkov telescope systems measure astrophysical $\gamma$-ray emission against a background of cosmic-ray induced air showers. The subtraction of this background is a major challenge for the extraction of spectra and morphology of $\gamma$-ray sources.

Aims. The unprecedented sensitivity of the new generation of ground based very-high-energy $\gamma$-ray experiments such as HESS has lead to the discovery of many previously unknown extended sources. The analysis of such sources requires a range of different background modelling techniques. Here we describe some of the techniques that have been applied to data from the HESS instrument and compare their performance.

Methods. Each background model is introduced and discussed in terms of suitability for image generation or spectral analysis and possible caveats are mentioned.

Results. We show that there is not a single multi-purpose model, different models are appropriate for different tasks. To keep systematic uncertainties under control it is important to apply several models to the same data set and compare the results.
\end{abstract}

Key words. gamma rays: observations - methods: data analysis

\section{Introduction}

Ground based very-high-energy $\gamma$-ray telescope systems such as HESS (Hinton 2004), MAGIC (Lorenz 2004), VERITAS (Weekes et al. 2002) and CANGAROO-3 (Kubo et al. 2004) have greatly increased the sensitivity of the Atmospheric Cherenkov technique. However, these instruments can only reach their full potential if systematic effects are brought fully under control. A major challenge for experiments of this type is the subtraction of the background of non- $\gamma$-ray induced air showers. This background can be dramatically reduced using image-shape selection criteria, but cannot be removed completely. The background above a few hundred $\mathrm{GeV}$ is dominated by hadronic cosmic-ray showers, with cosmic-ray electrons increasingly important at low energies and after tight image selection cuts. The subtraction of this background is the main source of systematic errors and, if not done correctly, can even produce an artificial source.

For single telescope instruments (for example the pioneering Whipple observatory $10 \mathrm{~m}$ telescope (Weekes et al. 1989)) the classical approach to background subtraction was the $O N / O F F$ observing mode. In this mode observations (runs) centred on the target source are interspersed with equal-length observations of an empty field at equal zenith angle (typically a region offset in Right Ascension by 30 minutes). The background is assumed to be equal in the two runs, the difference between them provides a measurement of the $\gamma$-ray signal. A major drawback of this approach is that only half of the available dark time is spent ON-source. The wobble-mode pioneered by the HEGRA collaboration (Daum et al. 1997) avoids this problem by keeping the targeted source region in the field of view (FoV) at all times, with an alternating offset relative to the system's pointing direction (for point sources typically $\pm 0.5^{\circ}$ in Declination). A background estimate ( $O F F$ data) for the source region ( $O N$ data) can then be derived from a region on the opposite side of the FoV from the same run as the $O N$ data.

Serendipitous detections of non-targeted sources such as HESS J1303-631 (Aharonian et al. 2005a), particularly in observations close to the Galactic plane, demonstrate the need for background models that provide background estimates for the whole FoV of an instrument. Any systematic survey of a whole sky region also requires such models. The importance of surveys has been demonstrated by the recent discovery of more than ten new $\gamma$-ray sources in a survey of the inner Galaxy with HESS (Aharonian et al. 2005b, 2006a). Moreover, extended $\gamma$-ray sources such as RX J1713.7-3946 (Aharonian et al. 2004, 2006b) and RX J0852.0-4622 (Vela Junior) (Aharonian et al. 2005c) present additional difficulties for background subtraction. They require correct background modelling over a region of the sky that is substantially larger than the source itself.

Many different approaches to background modelling are possible and have been applied in the analysis of data from the HESS instrument. These models have different strengths and weaknesses and it is usually desirable to apply several of them in the analysis of any $\gamma$-ray source to get a handle on the systematic uncertainties connected to the background determination. In this paper we aim to describe some of these approaches. While we will be using HESS data as the basis for our examples it should be noted that the techniques described here apply generally to VHE $\gamma$-ray instruments. Where necessary we will point out 
features that are specific to the analysis applied or to HESS and its large FoV.

An outline of this paper is as follows: we begin in Sect. 2 with a general introduction of background modelling and investigate in detail the properties of the detector acceptance ${ }^{1}$, as it is a key starting point for almost all background models. We then move on to a description of individual background models (Sect. 3). In Sect. 4 we compare the results of different approaches applied to particular data examples. In Sect. 5, the impact of bright stars on observations with atmospheric Cherenkov telescopes is demonstrated. Section 6 summarises the strengths and weaknesses of the different background models and describes to which purpose they are best suited.

\section{Cosmic-ray system acceptance after $\gamma$-ray cuts}

With cuts on image-shape parameters the cosmic-ray background can be reduced drastically (for a stereoscopic instrument with $100 \mathrm{GeV}$ threshold the rejection factor is roughly 100 , resulting in a signal-to-background ratio for a strong point source like the Crab nebula on the order of 1:1 (Aharonian et al. $2006 \mathrm{c})$ ). The remaining background of $\gamma$-ray-like events ${ }^{2}$ must be estimated to derive the significance of any possible $\gamma$-ray signal. Given a number of counts $N_{\text {on }}$ in a test region, and $N_{\text {off }}$ counts in a background control region, the $\gamma$-ray excess is defined as

$N_{\text {excess }}=N_{\text {on }}-\alpha N_{\text {off }}$.

The parameter $\alpha$ is a normalisation factor which accounts for differences between the test region and the background control region. The differences concern the solid angle, exposure time, zenith angle, and the detector acceptance for $\gamma$-ray-like cosmicray events ${ }^{3}$. In general, $\alpha$ is the ratio of the acceptance-weighted exposure integrated in time and angular space over the signal and background region (usually referred to as the $O N$ and $O F F$ regions). It can generally be defined as:

$\alpha=\frac{\int_{\text {on }} A_{\text {on }}^{\gamma}\left(\psi_{x}, \psi_{y}, \phi_{\mathrm{z}}, E, t\right) \mathrm{d} \psi_{x} \mathrm{~d} \psi_{y} \mathrm{~d} \phi_{\mathrm{z}} \mathrm{d} E \mathrm{~d} t}{\int_{\text {off }} A_{\text {off }}^{\gamma}\left(\psi_{x}, \psi_{y}, \phi_{\mathrm{z}}, E, t\right) \mathrm{d} \psi_{x} \mathrm{~d} \psi_{y} \mathrm{~d} \phi_{\mathrm{z}} \mathrm{d} E \mathrm{~d} t}$.

$A_{\text {on,off }}^{\gamma}$ is the detector acceptance for $\gamma$-ray-like events and depends on the position $\left(\psi_{x}, \psi_{y}\right)$ in the FoV, the event energy, and the zenith angle $\phi_{\mathrm{z}}$ of observations. Moreover, different exposure times $t$ for $O N$ and $O F F$ region have to be taken into account. If $O N$ and $O F F$ region are different in size and shape, the integration of the detector acceptance in Eq. (2) must take this into account. Note that the definition of $\alpha$ is the most general one and explicit analyses might require adaptations of the above formula. For example, in case of a morphological analysis, one would clearly not integrate over $\left(\psi_{x}, \psi_{y}\right)$, but calculate $A$ for the given position in the FoV. On the other hand, in case of a spectral analysis, differential energy spectra are derived, and hence the integration over $E$ is omitted.

Given a number of $O N$ and $O F F$ counts and $\alpha$, the statistical significance $(S)$ of the excess is typically calculated following

\footnotetext{
1 The term detector acceptance is used synonymously with (telescope) system acceptance throughout this paper.

2 The term $\gamma$-ray-like events refers to cosmic-ray background events that pass all selection cuts (cut values are optimised by means of $\gamma$-ray simulations).

${ }^{3}$ The exact definition of the term acceptance will be given further down.
}

the prescription of Li \& Ma (1983, Eq. (17)). The task of a background model is to provide the quantities $N_{\text {off }}$ and $\alpha$. A choice of background regions such that $\alpha \ll 1$ (achievable, e.g., by choosing much larger OFF than $\mathrm{ON}$ regions) results generally in higher statistical significance, because background fluctuations are reduced, but may also result in increased systematic errors. The principle difficulty in deriving a background estimate is the determination of the correct value of $\alpha$. Since proper control over the detector acceptance is crucial for this purpose, we investigate below the acceptance of HESS.

The detector acceptance specifies the probability of accepting a $\gamma$-ray-like event reconstructed at a certain position in the telescope system's FoV and with a certain energy. It is determined using cosmic-ray events that triggered the telescope array and survive selection cuts. For most background models some knowledge of the system acceptance is required to generate an image of $\gamma$-ray excess events or calculate significances of arbitrary positions in the FoV. In general the acceptance depends on:

- the position in the FoV, particularly the distance to the telescope-system pointing direction (often simply referred to as system centre);

- the zenith and azimuth angle of observations, due to the influence of the Earth's magnetic field on the shower development in the atmosphere and the rotational asymmetry of the telescope system;

- the reconstructed primary energy;

- the time of observation, due to possible changes in the system configuration and aging of mirrors;

- the sky coordinates viewed, due to the night-sky background light level.

In most cases it is a reasonable assumption that the acceptance is radially symmetric, that is, only dependent on the radial distance to the system centre, rotationally symmetric otherwise (the validity of this assumption is discussed later). It is generated in a one-dimensional fashion as the number of background events as a function of the (squared) angular distance between reconstructed event direction and system pointing direction. It can either be determined on a run-by-run basis from the data set under analysis or be extracted from observations without significant $\gamma$-ray emission in the FoV ( $O F F$ runs). In the latter case it is assumed that the system acceptance is identical for the $O N$ and $O F F$ runs. In the former case one may face two problems, $\gamma$-ray contamination by a source, and lack of statistics. For a typical HESS data run lasting 28 minutes, recorded at moderate zenith angles, the number of events after $\gamma$-ray selection cuts (available for the determination of the acceptance) is $O\left(10^{4}\right)$ with cuts for spectral analysis, and as low as $O\left(10^{3}\right)$ with cuts for morphology studies (see below for a description of the analysis cuts).

Here we use $220 \mathrm{~h}$ of HESS observations without significant $\gamma$-ray sources in the FoV to obtain a model of the radial system acceptance. These reference observations are sub-divided into zenith-angle bands. Events passing $\gamma$-ray cuts (i.e. $\gamma$-raylike background events) are then binned according to the squared angular distance between the reconstructed event direction and the system's pointing direction. Figure 1 illustrates the dependence of the acceptance on the zenith angle of observations and analysis cuts. The shallow central peak and rapid decline towards larger distances stems from the analysis based on image (Hillas) parameters (Hillas 1985), where a cut on the distance between image centre-of-gravity and the camera centre is applied to avoid truncation effects at the camera edge. Due to the finite camera size, edge effects are inherent and will always appear independent of the exact analysis applied. 

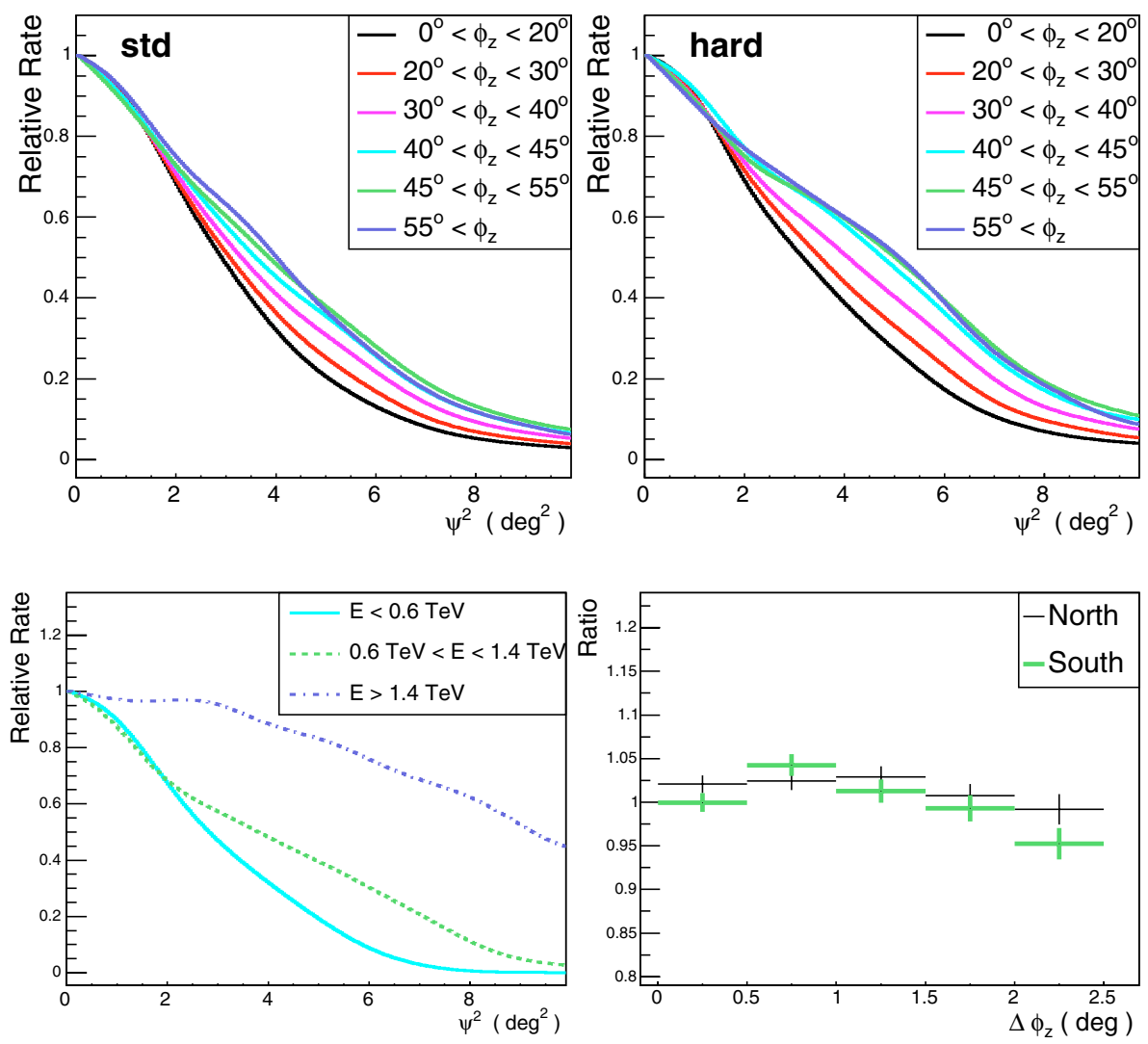

Fig. 1. The variation of the radial system acceptance function with zenith angle for two different sets of cuts. The distributions show the squared angular distance $\psi^{2}$ between reconstructed event directions and pointing direction of the telescope system generated from $O F F$-source data. The curves are generated by smoothing the one-dimensional acceptance histograms. They are arbitrarily normalised to 1 at the system pointing direction, corresponding to $\psi^{2}=0$. Left: the standard loose set of cuts (labelled $s t d$ ) is shown. It is mainly used for the determination of $\gamma$-ray spectra. Right: the hard cuts employing a larger cut on the minimum image amplitude are shown. This configuration is typically used for morphology studies.

Fig. 2. Left: the energy dependence of the system acceptance is demonstrated. For the three different energy bands shown, the shape of the acceptance broadens dramatically with increasing energy. Right: plot to test for a zenith-angle dependent FoV gradient (note the change of scales compared to the left-hand side). For $O F F$ data taken at zenith angles of $40^{\circ}$ to $45^{\circ}$, the data are split according to the azimuth of the observations: to the north (black crosses) and to the south (green crosses).
As can be seen from Fig. $1,2^{\circ}$ away from the system centre, the $\gamma$-ray acceptance at moderate zenith angles decreases to $30 \%-60 \%$ of the peak value, depending on analysis cuts. In addition a smooth variation with zenith angle is apparent. With increasing zenith angle, the system acceptance broadens, an increasing fraction of events with directions further away from the system pointing direction is detected. This is a direct consequence of the fact that with increasing zenith angle the shower maximum is increasingly further away from the telescope system causing an increase of the area illuminated on ground by the Cherenkov light pool and hence an enlarged phase space for events with large inclination angles. When comparing the average curve for any given zenith-angle band to the radial acceptance in different fields of view, observed at the same altitude, the scatter is relatively small, less than $3 \%$ within $1^{\circ}$ of the observation position and less than $10 \%$ out to $3^{\circ}$. It is therefore justifiable to use $O F F$ data taken in different fields of view to determine a model of the system acceptance.

The influence of analysis cuts is also apparent in Fig. 1. The two sets of cuts used throughout this paper are labelled std and hard. The first set includes a cut on the minimum amplitude of each camera image ${ }^{4}$ at 80 photo-electrons (p.e.) and is optimised for the determination of source spectra. The second set uses a cut on the image amplitude at 200 p.e., and provides better background suppression and superior angular resolution. It is therefore normally used for source searches and image generation (more detailed descriptions of the HESS analysis techniques may be found in Aharonian et al. (2005d) and Aharonian et al. (2006b)). The larger cut on the minimum image size results in curves which exhibit a generally less pronounced peak and a less

${ }^{4}$ The amplitude of a camera image is defined as the sum of all pixel intensities after a suitable image-cleaning procedure. This amplitude is also called image size. rapid decline towards large distances. There is an increased fraction of events with large inclination angles with respect to the system pointing direction.

The azimuth dependence of the radial system acceptance is negligible here: when sub-dividing data taken in a narrow zenithangle band into azimuth bins (say North, East, South, and West), only marginal differences occur at the few-percent level. The energy dependence of the acceptance is much stronger, greatly complicating the use of background models that require an acceptance correction for spectral analysis. This is illustrated in Fig. 2 (left) where the energy dependence for a zenith angle range from $0^{\circ}$ to $20^{\circ}$ is plotted. The curves shown correspond to three different energy bands, $E<0.6 \mathrm{TeV}, 0.6 \mathrm{TeV}<E<$ 1.4 TeV, and $1.4 \mathrm{TeV}<E$. For relatively small energies the acceptance declines rapidly with increasing offset. For large energies the shape is completely different. High-energy showers have a larger Cherenkov photon density, therefore the telescope system is triggered by showers with larger impact parameters by the tail of photons beyond the rim of the Cherenkov light pool. Therefore, as already mentioned, more events with large angular offsets start to trigger the array. In effect, for energies beyond $1.4 \mathrm{TeV}$, the acceptance is almost flat out to a distance of $2^{\circ}$ from the system pointing direction.

As previously mentioned, in most cases the system acceptance is assumed to be radially symmetric. The most intuitive cause of deviations from radial symmetry is a zenith-angle dependent linear gradient across the FoV. The larger the zenith angle of observation, the larger the effective energy threshold of the system because of the decreasing Cherenkov photon density. Since the energy spectrum of the cosmic-ray background is rather steep, the trigger rate, and thus the event rate, of the system decreases smoothly with increasing zenith angle (Funk et al. 2004). Hence, in the HESS FoV of $\approx 5^{\circ}$ significant variations of the system acceptance along the zenith axis may occur, 

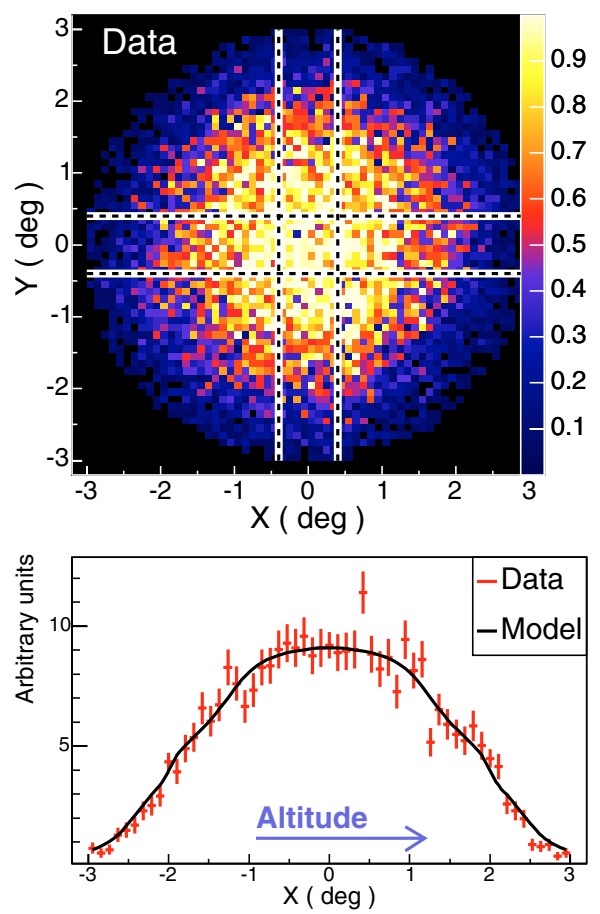
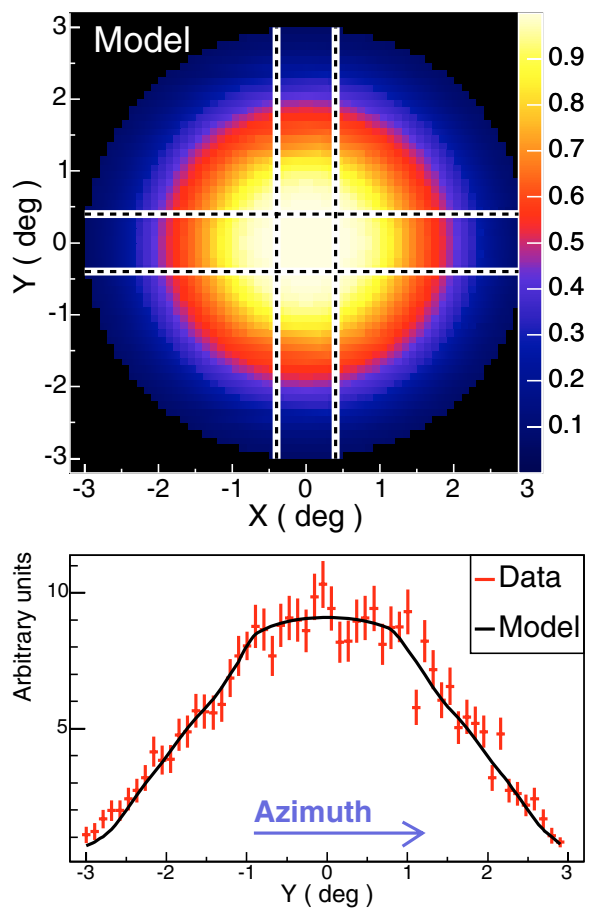

Fig. 3. Plots of the system acceptance are shown for HESS observations of the supernova remnant SN 1006 (Aharonian et al. $2005 \mathrm{e}$ ), compared to the radially symmetric model acceptance determined from $O F F$ runs. Upper panel: plots labelled "Data" and "Model" are the (arbitrarily normalised) acceptance maps determined in the nominal coordinate system on a run-by-run basis. Positive $x$-direction corresponds to positive altitude, positive $y$-direction to positive azimuth. The parallel horizontal and vertical lines define bands $\left(0.8^{\circ}\right.$ wide $)$ used to produce slices for a one-dimensional comparison, shown in the lower panel: the two plots show projections along $x$ and $y$ through data (red crosses) and model map (black lines) within the thick bands indicated in the upper panel. and indeed such variations are observed. Depending on zenith angle the peak system acceptance extracted from $O F F$ runs does not coincide with the nominal system pointing direction. It is found to be slightly shifted towards smaller zenith angles, away from the pointing direction. To account for this effect, the zenithangle dependence of that shift is determined: for small zenith angles $\left(\sim 10^{\circ}\right)$ the shift is negligible $\left(<0.01^{\circ}\right)$, at moderate $30^{\circ}$ it is on the order of $0.03^{\circ}$ and exceeds $0.13^{\circ}$ for very large zenith angles beyond $60^{\circ}$. A parametrisation is used to correct the nominal centre of the system pointing direction in each $O F F$ run. Figure 2 (right) explores remaining deviations from radial symmetry along the zenith axis. For this purpose, for $O F F$ data taken at zenith angles of $40^{\circ}$ to $45^{\circ}$, the symmetry of the system acceptance along an axis running parallel to the zenith direction is investigated: we fill all events into a histogram in bins of zenith-angle difference $\Delta \phi_{\mathrm{z}}$ between the event direction and the system pointing direction. To test the symmetry with respect to the pointing direction, the ratio is calculated from the number of events in bins on either side of the central $\left(\Delta \phi_{\mathrm{z}}=0\right)$-bin, equally far away from this bin. The resulting distribution is shown in Fig. 2 (right) as a function of $\left|\Delta \phi_{\mathrm{z}}\right|$ for two azimuthal bands (north and south) to search for effects related to the Earth's magnetic field (note that all corrections accounting for mis-pointing of the telescopes and for zenith-angle dependent trigger rates are included now). If there was no zenith-angle dependence, the ratio would be 1 for the whole FoV. There seems to be a residual distortion of the system acceptance along the zenith axis, in the direction one would expect from the trigger-rate variation. Larger zenith angles have smaller event numbers, indicating a slight under-correction of this effect. However, remaining deviations are estimated to be less than $5 \%$. Within statistics, there is no North-South effect apparent, the event-ratio distributions are in reasonable agreement with each other.

The validity of the simplifying assumption that the system acceptance is radially symmetric can be verified with data. For that purpose such a symmetric model acceptance derived from $O F F$ data can be compared to the acceptance of a single data set without $\gamma$-ray source, for example the HESS data from the
2003-2004 observation campaign of SN 1006 (Aharonian et al. 2005e) (which shows no evidence of $\gamma$-ray emission). Reconstructed directions of $\gamma$-ray-like events are plotted with respect to the telescope-system pointing direction in the "altitudeazimuth" coordinate system (also referred to as nominal coordinate system). Accumulating events from different data runs one obtains in this way a sample two-dimensional $\gamma$-ray acceptance map. This can be compared to a model acceptance map derived by choosing, for each run, the one-dimensional radial acceptance for the corresponding zenith angle (cf. Fig. 1). The acceptance is then rotated in the nominal system and accumulated for all runs yielding an acceptance model which can be compared to the system acceptance deduced from the data set. The result, derived from $6.3 \mathrm{~h}$ (after dead-time correction) of (4-telescope) HESS observations of SN 1006, is shown in Fig. 3. There is general agreement between data and model acceptance. Remaining differences are at the few percent level.

\section{Background models}

Having discussed the system acceptance function, which is crucial for all background models, we now return to the principal task of a background model: to provide estimates of $N_{\text {off }}$ and $\alpha$ (Eqs. (1) and (2)). Various background estimation techniques are described below.

\subsection{Ring background}

A method that is robust in the face of linear gradients in arbitrary directions is the ring-background model. In this model a ring around a trial source position (in celestial coordinates) is used to provide a background estimate. This is applicable to any point in the FoV. The parameter $\alpha$ is approximately the ratio of the solid angle of the ring (of typical radius $0.5^{\circ}$ ) to the trial source region $\left(\Omega_{\mathrm{on}} / \Omega_{\text {off }}\right)$, and is typically chosen to be $\sim 1 / 7$. However, within the ring the acceptance cannot be assumed to be constant, since the ring covers areas with different offsets from the observation position. Therefore an acceptance correction 

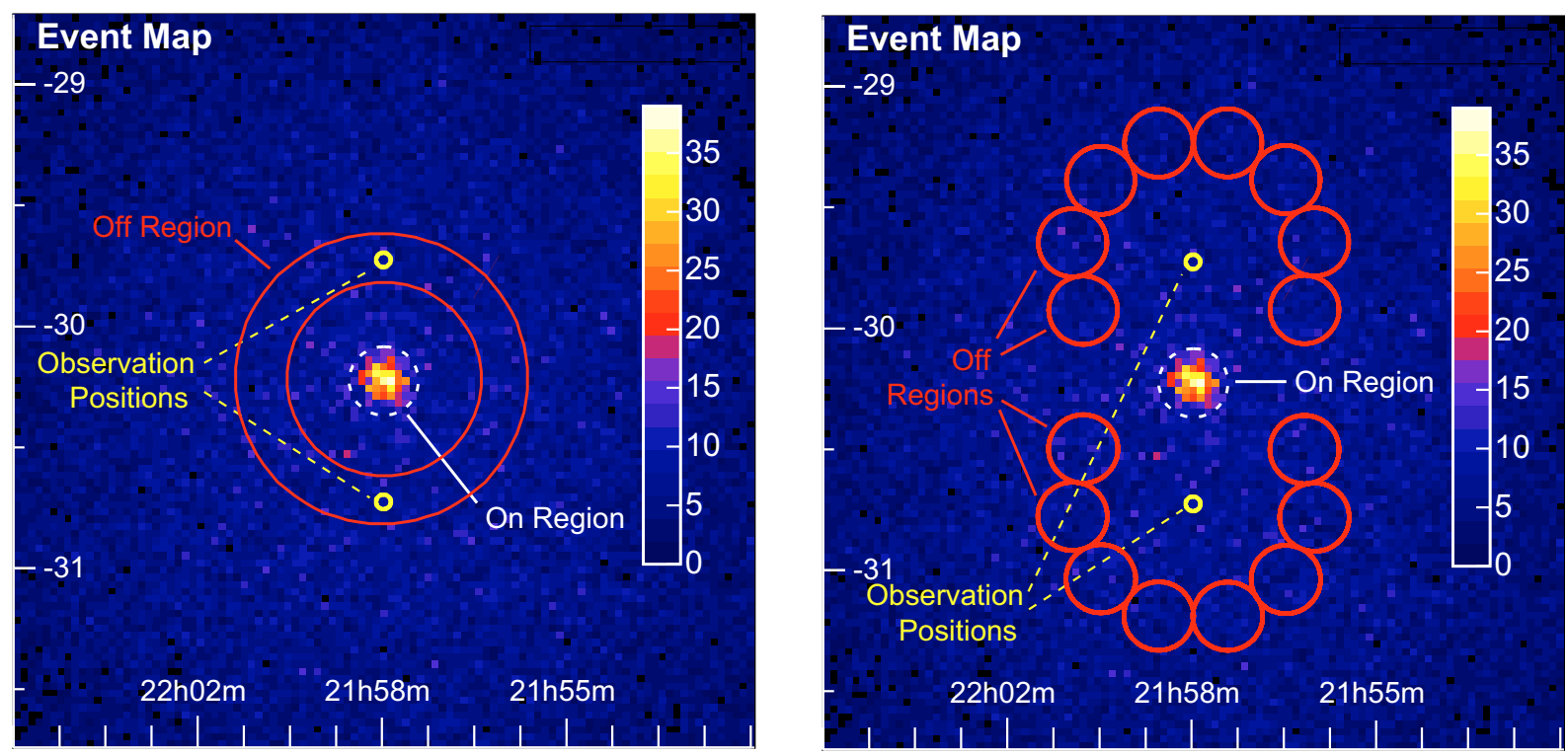

Fig. 4. Count map of $\gamma$-ray-like events from $5 \mathrm{~h}$ of HESS observations of the active galaxy PKS 2155-304 (Aharonian et al. 2005d). Note that the data were taken in wobble mode around the target position with alternating offsets of $\pm 0.5^{\circ}$ in declination. The ring- (left) and reflected-region(right) background models are illustrated schematically.

function must be used in the determination of the normalisation $\alpha$ for each position on the ring. The ring-background method is illustrated schematically in Fig. 4 (left).

\subsection{Reflected-region background}

The reflected-region-background model was originally developed for wobble observations (Aharonian et al. 2001, 2006c), but can be applied to any part of the FoV displaced from the observation position. For each trial source position a ring of $n_{\mathrm{off}}$ $O F F$ regions is used (see Fig. 4 (right)). Each $O F F$ region is the same size and shape as the $O N$ region and has equal offset to the observation position (note that here the ring is centred on the observation position, while for the ring background technique the ring is centred on the trial source position). The method is called reflected-region method because the $O N$ region is reflected with respect to the FoV centre to obtain one $O F F$ region. In the general case as many reflected $O F F$ regions as possible are then fit into the ring whilst avoiding the area close to the trial position to prevent contamination of the background estimate by misreconstructed $\gamma$-rays. Due to the equal offset of $O N$ and $O F F$ regions from the pointing direction of the system, no radial acceptance correction is required with this method and $\alpha$ is just $1 / n_{\text {off }}$. This is particularly helpful for spectral analysis where an energy-dependent radial acceptance function would otherwise be required. In case the $\gamma$-ray source was observed under a large range of offset angles with respect to the system pointing direction, for example as part of a sky survey, the normalisation $\alpha$ might differ substantially from run to run. In this case, a suitable averaging procedure has to be applied to both nominator and denominator of Eq. (2): the exposure measure is weighted by a factor taking account of the offset of the source from the pointing direction (this factor might be calculated as the ratio of the $\gamma$-ray acceptance at the offset of the run to the acceptance at a reference offset).

We note that the tracking-ratio method (Kerrick et al. 1995), first applied to the data of the Whipple observatory $10 \mathrm{~m}$ telescope, is somewhat similar to the reflected-region method. In that approach, the source or signal region is defined by images pointing towards the putative source location, the background level is estimated from images pointing away from the source direction. This background model is only suitable for singletelescope data and is therefore not investigated here.

\subsection{Template background}

The template-background model was first developed for the HEGRA instrument and is described in Rowell (2003). This method uses background events displaced in image-shape parameter space rather than in angular space. A subset of events failing $\gamma$-ray selection cuts are taken as indicative of the local background level. The approach is demonstrated in Fig. 5 (left), where the distribution of the mean reduced scaled width $(M R S W)$ is shown for $\gamma$-ray and proton simulations (the separation potential of the MRSW is clearly seen; it is frequently used for background suppression in HESS analyses (Aharonian et al. 2005d)). Events falling into the Signal regime are considered $\gamma$-ray-like events and are taken as $O N$ counts, events falling into the background regime $(3.5 \sigma \leq \mathrm{MRSW} \leq 8 \sigma)$ are considered cosmic-ray-like events and are taken as $O F F$ counts. The normalisation $\alpha$ is calculated as the number of events in the Signal regime, excluding the source region, divided by the number of events in the Background regime. A correction factor depending on the position in the FoV has to be applied to $\alpha$ since the system responds differently to the cosmic-ray-like than to the $\gamma$-ray-like events. Therefore, an additional radial acceptance curve for the Background regime has to be determined. This cosmic-ray acceptance curve depends on the choice of Background regime. In practice it turns out that the system acceptance becomes very different from the $\gamma$-ray acceptance if Signal and Background regime are too far apart. This is undesirable because the necessary correction factor would vary strongly within a FoV, potentially increasing systematic uncertainties. The choice of Background regime is thus a compromise between good separation from the Signal regime and small $\alpha$ (i.e. reasonable event statistics), and obtaining a background system acceptance function which does not differ substantially from the $\gamma$-ray acceptance. For the particular choice of Background 

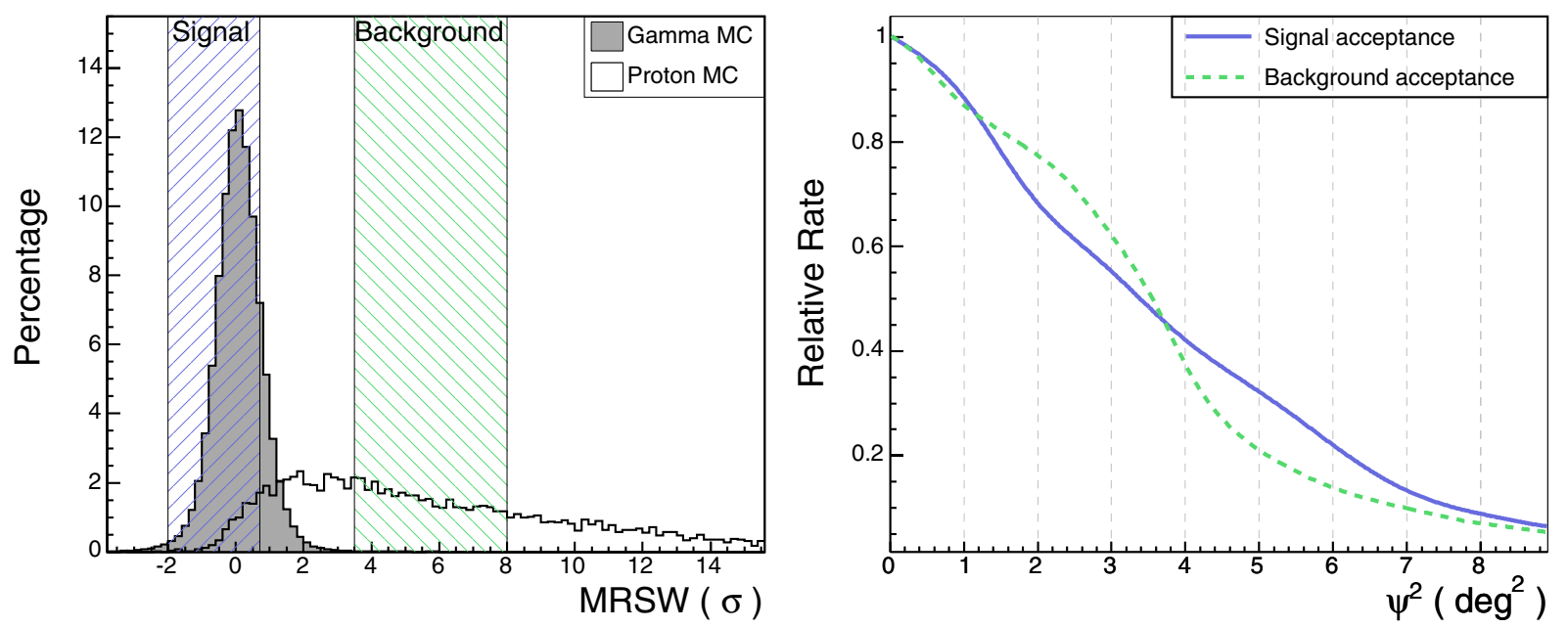

Fig. 5. Illustration of the template-background model. Left: distribution of the mean reduced scaled width (MRSW) from $\gamma$-ray as compared to proton simulations. This parameter is defined as the deviation in units of standard deviation of the measured image width from the simulated expectation value, averaged over all telescopes participating in an event. Right: system acceptances for the Signal and Background regimes as indicated in the legend. The distributions are generated from $O F F$ runs, the statistical error bars are negligible. The Background acceptance is normalised to the area of the Signal acceptance in the central $1.5^{\circ}$.

regime applied here, the difference of the two system acceptance curves can be seen in Fig. 5 (right). In the central part, for event directions close to the system pointing direction, the two curves are very similar. For offset angles larger than $\sim 1.5^{\circ}$ pronounced differences occur. The ratio of the $\gamma$-ray and cosmic-ray acceptances, which is required to determine $\alpha$, will not be constant over the FoV. In the case of long observation times, differences in the system acceptance are a sizable effect and a potential sources of a systematic error.

The template method has the advantage that the background is determined in the same region as the signal and hence any localised problem, for example due to a bright star, will affect both signal and background. Whether the effect is equal for both and therefore cancels out depends on the choice of the Background regime and has to be checked from case to case. A drawback of this method is that exact knowledge, not only of the Signal, but also the Background acceptance is required, potentially increasing systematic uncertainties related to the modelling of the system acceptance.

Another method that has been applied to HESS data is the weighting method which is related to the template background. Signal and background are estimated simultaneously from the same portion of the sky. Events with directions associated with a certain sky bin are assigned two weights, one for the assumption that it is a signal event, one that it is a background event. Subtraction of the accumulated bin content yields the $\gamma$-ray excess. This approach is not pursued further, but is described in detail elsewhere (Lemoine-Goumard \& Degrange 2005).

\subsection{Field-of-view background}

For the field-of-view-background model, the entire field (excluding regions of known $\gamma$-ray emission) is used for the normalisation of an acceptance model to the data and the normalisation $\alpha$ approaches zero. The acceptance model is derived from the set of $O F F$ runs mentioned above. Given an observation at a certain zenith angle, a model background map is created by rotating the radial acceptance curve (cf. Fig. 1) of the corresponding zenith angle band. The advantage of this model is that it can be readily applied to extended sources and results in the highest possible statistical significance. However, the method is sensitive to deviations of the true system acceptance from the model applied.

\subsection{Classical ON/OFF background}

As mentioned earlier, in traditional $O N / O F F$ mode twice the observing time is required for each source, providing a strong disincentive for this approach to background modelling. Nevertheless, the $O N / O F F$ mode has a powerful advantage in that no assumption is made for the system acceptance, except that it is the same in both exposures. As the $O N$ and $O F F$ runs have identical pointing direction in azimuth and elevation, the only assumption made is that the acceptance is not dependent on conditions fixed in celestial coordinates, such as stars and nightsky background light. This advantage motivates a modified form of $O N / O F F$ analysis that has been applied to HESS data. A sizable fraction of the fields observed with the HESS instrument contain no significant $\gamma$-ray signal. These data can be used as an archive of $O F F$ data. For a given set of $O N$ runs, a set of $O F F$ runs matching in zenith angle is selected from the archive. The normalisation, $\alpha$, between $O N$ and $O F F$ runs is deduced from the total event numbers in the two runs, excluding the nominal $\gamma$-ray source region.

\section{Model comparison}

A satisfactory background model must meet one main criterion: when applied to many trial positions it should produce a normal distribution of significance for an empty field. To test this, four of the background models described here have been applied to the HESS data of SN 1006, which (as already mentioned) shows no evidence for $\gamma$-ray emission (Aharonian et al. 2005e). Figure 6 (left) compares significance maps of this one field derived using four different background models. The hard cuts have been applied and the significance at each trial sky position was calculated integrating $O N$ events within a circle of $0.1^{\circ}$ radius (note that due to this integration, the bins of the significance maps are correlated). A map of absolute significance is shown only for the field-of-view model. To ease comparison, for other models the difference in significance to the reference 

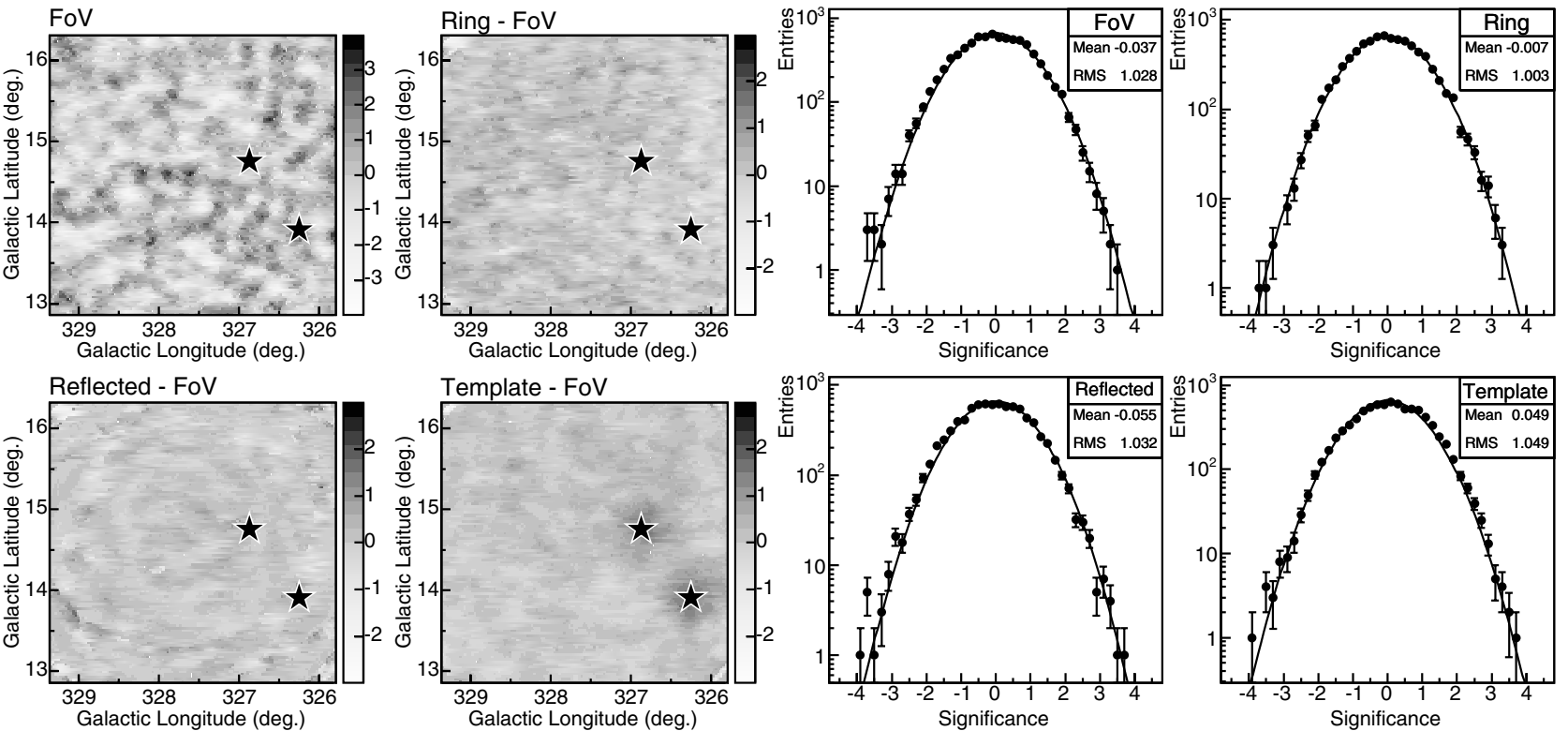

Fig. 6. Left: maps of statistical significance for the field around the supernova remnant SN 1006 derived using four different background models. $6.3 \mathrm{~h}$ of 4-telescope HESS data without significant $\gamma$-ray signal are used. The black stars in each field mark the position of bright $\left(m_{B}=2.5\right.$ and $\left.m_{B}=2.9\right)$ stars. Further descriptions are given in the main text. Right: distributions of significance in the FoV around SN 1006 for the different models. The solid black curve illustrates the expected normal Gaussian distribution. As can be seen, deviations from the expected behaviour are at the less than $1 \%$ level.

field-of-view-model is plotted. The maps show satisfactory agreement with each other on the $1 \sigma$-level, within the expected statistical fluctuations. The difference maps are roughly constant throughout the field, with the only exception being the regions close to two bright stars in the lower right of the FoV. As is described in more detail below, bright stars cause a reduction in the local rate of events, producing a dip in all significance maps except that derived using the template-background model, in which case the bright stars influence also the background estimate. For the particular choice of background regime employed here, the template model slightly over-corrects for the dip in the $\gamma$-ray acceptance, producing a positive significance at the star positions.

Figure 6 (right) shows the distributions of significance values of each trial source position for the maps shown in Fig. 6 (left). The regions close to the two bright stars have been excluded. The distributions show satisfactory agreement with the expected normal Gaussian. We note that deviations on the mean significance slightly larger than $1 / \sqrt{N_{\text {trials }}}$ are expected due to the correlations between the signal and background estimates in neighbouring positions. These distributions show that, at least for this FoV, the systematic error on the assignment of a statistical significance to the signal at a given position is at the $<0.1 \sigma$ level. Furthermore, the agreement between different models suggests that under normal circumstances they all provide valid background estimates with fluctuations at or close to the expected Poisson behaviour.

Figure 7 compares a $\gamma$-ray count map of the field around the supernova remnant RX J1713.7-3946 to three different background model maps. The count map is generated from HESS data from 2004 (Aharonian et al. 2006b) with four observation positions, all offset by $0.7^{\circ}$ from the centre of the remnant. In each observation run, the usable range of the FoV is restricted to the central $2^{\circ}$ around the observation position causing edges in acceptance when overlaying data from different observations. The background models are normalised by $\alpha$ and shown on the same scale. It is apparent that the different models have different levels of statistical fluctuations. For the template-background map the statistics are reasonably good, $\alpha \approx 1 / 14$ when choosing a background regime of $3.5 \sigma \leq$ MRSW $\leq 8 \sigma$ (cf. Fig. 5) and hard cuts. $\alpha$ is practically zero in case of the field-of-viewbackground model, statistical fluctuations are negligible, consequently the background map is very smooth. The $O F F$-data map (from the $O N / O F F$ analysis) has the largest $\alpha(\approx 1)$, and statistical fluctuations are at a considerable level resulting in a comparatively low statistical significance of the signal. For illustration contour lines are overlaid on all four sky images in the figure. Apart from differing event statistics, the three background models are in good agreement both in terms of shape and absolute level. They clearly provide an appropriate description of the background of the $\gamma$-ray count map shown in Fig. 7 a).

A systematic comparison of the background level estimated by different models was performed for the whole HESS 2004 Galactic plane survey. Figure 4 of Aharonian et al. (2006a) shows the correlation between the background estimate for each grid point in the sky derived using ring- and templatebackground models. The correlation is close to linear over a large dynamic range. The spread is consistent with statistical fluctuations in $N_{\text {off }}$. The slope of the correlation is 1.007 and both background estimates are consistent within 1\% - see Aharonian et al. (2006a) for details.

Another demonstration of the validity of different background models is shown in Fig. 8. For two HESS data sets with significant $\gamma$-ray signal, slices along Right Ascension and encompassing the sources are shown. Overlaid, in both cases, are the field-of-view and template background models for these data sets. In both cases, at different regions in the sky, for an extended and a point-like $\gamma$-ray source, there is clearly a good match between both models and data in regions outside the $\gamma$ ray sources. Moreover, it is evident that the features of the $\gamma$ ray morphology of RX J1713.7-3946 after background subtraction are robust and remain unchanged when applying different background models. Note that this can also be seen in Fig. 6 of 

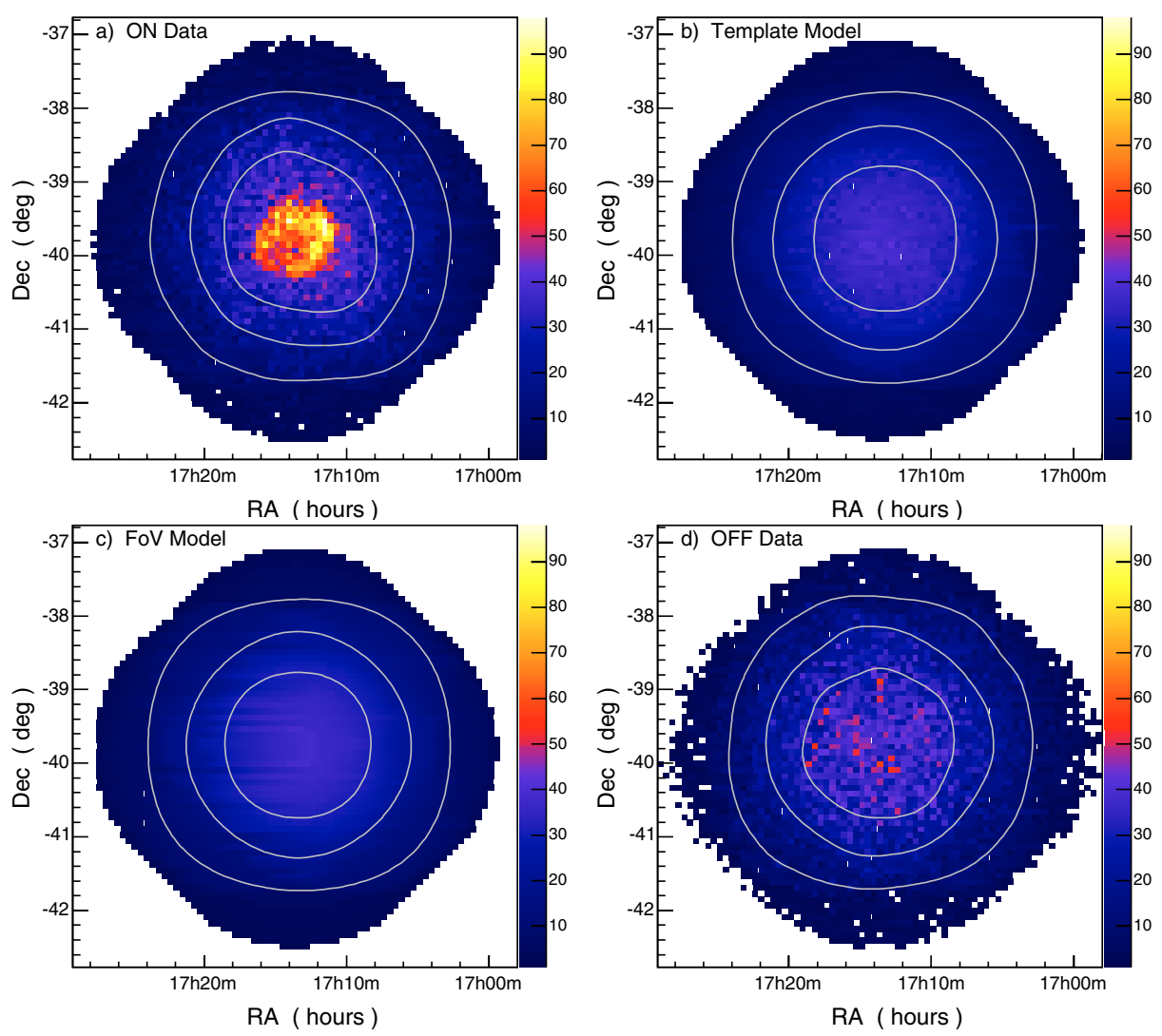

Fig. 7. Illustration of different background models for the case of the supernova remnant RX J1713.7-3946. Note that hard cuts have been applied here. a) Raw $\gamma$-ray count map generated from HESS 2004 data for the field around the remnant. b)-d) Normalised background maps derived using three different approaches (discussed in the main text). Overlaid on all four images are white contours for illustration. They are equally spaced at 10, 20, and 30 counts and are deduced from a Gaussian-smoothed version of the raw colour images to reduce the impact of statistical fluctuations.
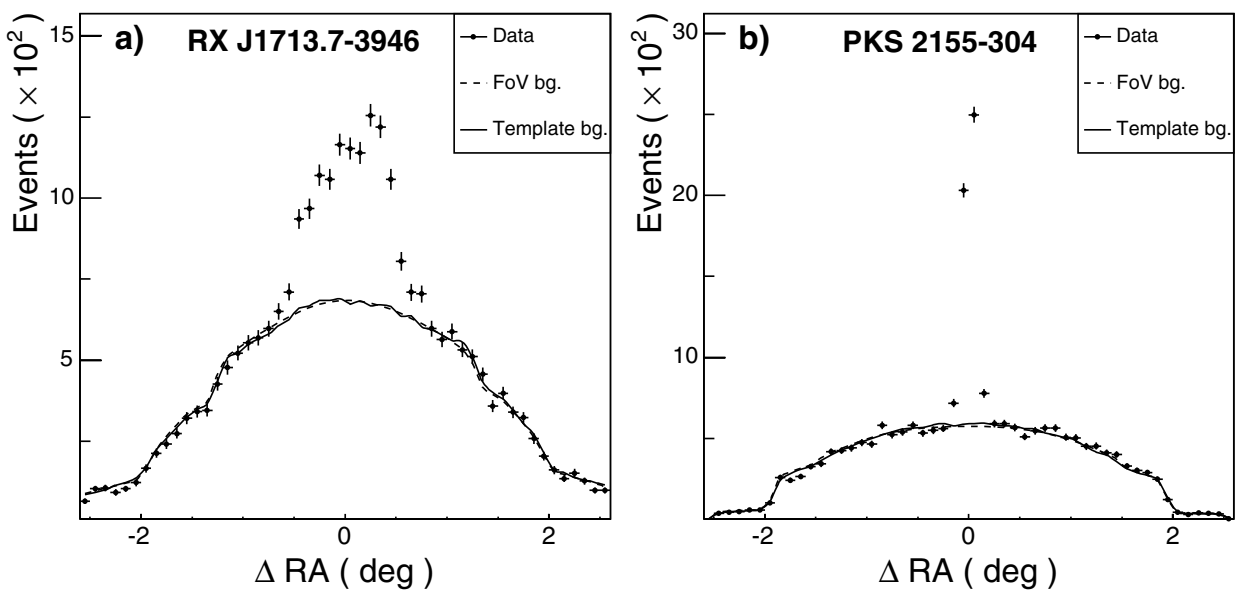

Fig. 8. Illustration of the agreement between data points and background model (solid and dashed lines). HESS data for the extended $\gamma$-ray source RX J1713.7-3946 a) are compared to PKS 2155-304, a point-like extra-galactic $\gamma$-ray source for which a high-statistics data set exists (Aharonian et al. 2005d) b). Slices along Right Ascension (RA) through the centre of (and fully encompassing) the sources, are shown. Two background models are shown, the field-of-view- (dashed line) and the template-background model (solid line). Note that the steps in the distributions are artifacts of the analysis: for each observation run, the usable range of the FoV is restricted, and the distributions are produced from different observation positions.

Aharonian et al. (2006b) which shows a linear correlation of $\gamma$ ray excess counts for the sky region around RX J1713.7-3946 for two different background subtraction methods, the weighting method (mentioned above and discussed elsewhere (LemoineGoumard \& Degrange 2005)) and the field-of-view method.

\section{Effect of stars}

All background estimates presented here rely on the homogeneity of the ( $\gamma$-ray or hadron) acceptance across the FoV. While detector acceptance inhomogeneities are typically of the order of $3 \%$ or less, they may reach higher values in special cases such 


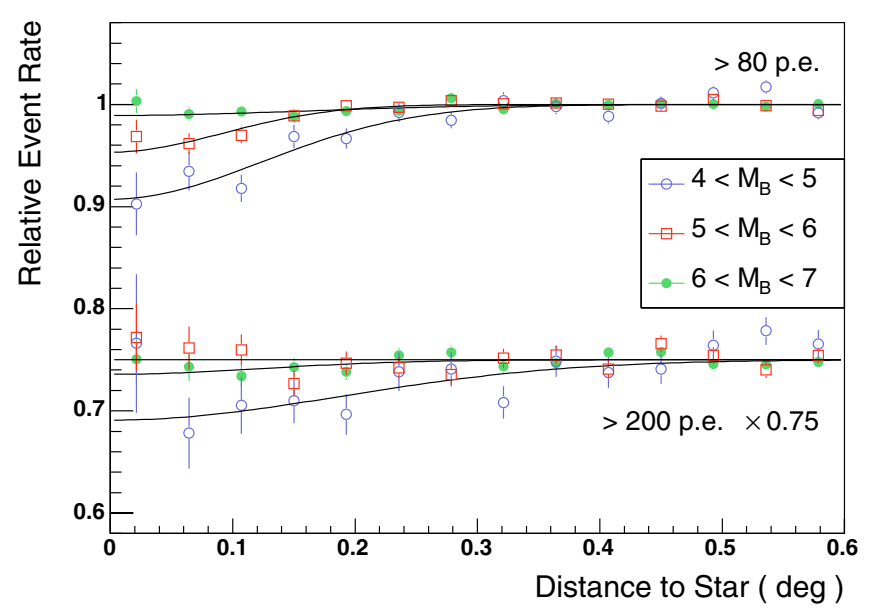

Fig. 9. Effect of bright stars on the event rate of the system for std and hard cuts (80 and 200 p.e., respectively). The relative event rate is shown as a function of the distance to bright stars for different $B$ magnitude bands. The 200 p.e. size-cut data set has been scaled by 0.75 for clarity.

as large zenith-angle observations or in the presence of strong sky-brightness variations (most frequently due to stars). Figure 9 shows as an example the event rate in arbitrary units, as a function of the distance to bright stars in the FoV, for different bands of stellar $B$-band magnitude. The curves were derived from the HESS Galactic Plane survey dataset, averaging over all stars in the respective magnitude interval. It can be seen that for stars with $B$-magnitude smaller than 5 , the event rate at the position of the star decreases noticeably. This effect can be explained as a consequence of the automatic switching off of pixels when DC illumination reaches potentially damaging levels. For events in which the shower core is located close to one of the telescopes, the event for that telescope will have a hole in the middle of its Cherenkov image due to pixels being switched off by the star. An image with a hole in the middle might a) be thrown away by the image cleaning, or b) fail the shape cuts. Therefore less events are reconstructed in the direction of the star. The effect increases with increasing brightness of the star. The histograms shown in Fig. 9 are derived using std cuts. For hard cuts the effect is less severe. This is expected, as the influence of individual pixels that are switched off is smaller for the larger images required with the increased image amplitude cut of 200 p.e. Thus, for the hard cuts, no significant dip at the position of stars can be seen in the magnitude 5 to 6 band. For the magnitude 4 to 5 band, a significant dip occurs for both sets of cuts. The magnitude of this effect is similar to that found previously for the HEGRA telescope array (Puehlhofer et al. 2003).

\section{Choice of model}

In the following we summarise the advantages and disadvantages of the available background models and list the analysis types they are most suited for. We start by listing properties of an ideal background model:

Contemporaneous: background events for a given data set should stem from a contemporaneous observation period to avoid incompatibilities due to ageing effects of the telescope system.

FoV position: background events should be accumulated at the same or a similar position in the FoV, meaning that the angular distance to the system pointing direction should be equal to the signal events (because of the angular dependence of the system acceptance, cf. Fig. 1).

Sky position: to assure a similar level of night-sky background light background events should be recorded from the same region of sky.

Event statistics: to reduce fluctuations, the event statistics of the background should be considerably larger than the signal one, implying a normalisation factor $\alpha \ll 1$.

Event type: signal and background events should be of the same type, from the same region in image-parameter phase space to assure a similar system response and reduce the importance of the correct system-acceptance model.

It is obviously impossible to fulfil all of these requirements with a single background model. Any choice can only be a compromise. It is therefore important to apply different models to a data set thereby cross-checking the results. Table 1 classifies the models discussed in Sect. 3 in terms of the properties listed above. The advantages and shortcomings of the different models are:

ring background: the model has the advantage of providing a conceptually simple prescription for the background determination. It is rather insensitive to deviations of the actual relative acceptance of the data set from the model acceptance function, as it only relies on the relative normalisation in a limited nearby area around the source bin in the FoV. Any linear gradients in the system acceptance are averaged out because of the summation on the ring. However, when testing larger source extensions, larger ring radii are required, demanding better accuracy for the relative acceptance correction across a larger portion of the FoV. For the determination of energy spectra, this method is disfavoured, since the acceptance curve changes with energy (as shown in Fig. 2 (left)). Any attempt to correct for this, e.g. by determining acceptance curves in energy bands, would introduce another source of systematic uncertainty. Additionally, the $O F F$ events have a different distribution of offsets from the centre of the FoV than the $O N$ events. Since the effective areas used for the spectral analysis depend on the camera offset, the different offset distribution introduces again an additional systematic uncertainty, even if one corrects for it. We note furthermore that in the case of several $\gamma$-ray sources or indications for sources in the FoV, a case which is becoming more common in Galactic observations with the current generation of experiments, the ring background suffers from additional systematic uncertainties, that is, possible $\gamma$-ray contamination: if the source is surrounded by several $3 \sigma$ spots, some of which might be actual so far undetected sources, they would all be included in the background estimate and lead to a systematic over-subtraction.

reflected-region background: it has the advantage that it is independent of the exact shape of the acceptance function. It simply relies on the assumption of radial symmetry of the acceptance. Additionally the distribution of offsets from the centre in the $O N$ and $O F F$ events is the same. This makes the model especially suited for the background estimation for energy spectra. However, this approach relies on a suitable observation strategy, it cannot be applied if the observation positions of a data set are within an extended source region, or, as mentioned above, in case of too many other $\gamma$ ray sources in the FoV. In this case one either ends up in a 
Table 1. Overview of the properties of the different background models described here. For each feature (described on page 1227) we quote if a given model fulfills $(Y)$ or fails $(N)$ this condition.

\begin{tabular}{lccccc}
\hline \hline & ring & reflected-region & template & field-of-view & ON/OFF \\
\hline Contemporaneous & $\mathrm{Y}$ & $\mathrm{Y}$ & $\mathrm{Y}$ & $\mathrm{Y}$ & $\mathrm{N}$ \\
FoV position & $\mathrm{N}$ & $\mathrm{Y}$ & $\mathrm{Y}$ & $\mathrm{N}$ & $\mathrm{Y}$ \\
Sky position & $\mathrm{N}$ & $\mathrm{N}$ & $\mathrm{Y}$ & $\mathrm{N}$ & $\mathrm{N}$ \\
Event statistics & $\mathrm{Y}$ & $\mathrm{Y}$ & $\mathrm{Y}$ & $\mathrm{Y}$ & $\mathrm{N}$ \\
Event type & $\mathrm{Y}$ & $\mathrm{Y}$ & $\mathrm{N}$ & $\mathrm{Y}$ & $\mathrm{Y}$ \\
\hline
\end{tabular}

${ }^{5}$ Unless the number of $O F F$ regions $n_{\text {off }}$ is small, e.g. for largely extended sources.

situation where it is not possible to define a reflected background region without overlap with an actual known source region, or, in case of close-by sources just below detection limit, one might obtain a $\gamma$-ray contaminated background estimate.

template background: this technique has the advantage that it is better suited to largely extended sources (which fill a sizable fraction of the experiment's FoV) than the ringbackground method as long as an acceptance model (say from $O F F$ data) is available. It is, however, sensitive to uncertainties in the relative acceptance determination between the Signal and Background regime across the FoV. Large differences of the system-acceptance functions in the two regimes potentially increase systematic uncertainties of the normalisation factor $\alpha$.

For the estimation of energy spectra the background estimate must consist of events with a similar distribution of estimated energies to the background events of the source region. The template model does not meet this criterion since the events in the background regime will differ in estimated energy from those in the signal regime. Also the problem of the acceptance curve changing with energy is present as in the case of the ring background but more severely since it applies to the signal as well as to the background regime.

field-of-view background: the model can readily be applied to any data set to investigate the source morphology. It is especially well suited for very extended sources that cover a large fraction of the FoV and yields the maximum possible signal significance since the normalisation factor $\alpha$ is practically zero. The caveat is that it is sensitive to deviations of the model from the true system acceptance. For example, pronounced night-sky brightness variations within a single field and data sets with unbalanced wobble observation offsets might cause distortions of the true system acceptance.

ON/OFF background: this classical approach is a robust method to perform cross-checks and explore systematic uncertainties of spectra of very extended sources. Its advantage is that it can be applied to any data set, independent of the source size and the observation strategy pursued for a given source (as opposed to the reflected-region approach, which relies on observation positions outside the source region). Its caveats are possible changes in the night-sky background level between $O N$ and $O F F$ data and comparatively small background event statistics.

To summarise, the best suited background estimation technique for the extraction of various aspects of the $\gamma$-ray signal are:

- Source detection: the ring-background model has in general fewest systematic biases for this purpose. Only in the case of busy sky fields which contain multiple potential $\gamma$-ray sources issues due to $\gamma$-ray contamination arise.
- Spectral analysis: a reflected-region background is most suitable due to the identical offset distribution of $O N$ and $O F F$ regions. For very extended sources, however, or for extended sources which have not been observed with sufficiently large wobble offset, or in case there are other $\gamma$-ray sources in the FoV and one cannot define background regions, the $O N / O F F$-background model is the only appropriate one.

- Morphology of very extended sources: the field-of-view model provides an effective way to investigate the morphology of sources too large to be effectively handled by other methods.

\section{Optimal wobble offset}

Besides choosing a background model best suited for a given source and the analysis task, the optimal observation strategy needs to be considered. It is governed by the source properties and the preferred background model to be applied. To derive a background estimate and extract a signal from a single dataset it is normally necessary to observe a potential source with an offset with respect to the pointing direction of the system. For a given background model and for known radial system acceptance, the optimum observation (or wobble) offset $\Theta_{\text {opt }}$ can be calculated. This offset is defined as the one which maximises the significance per unit observation time $(S / \sqrt{t})$ of the source. For ring and template backgrounds $\Theta_{\mathrm{opt}}=0$, but this is undesirable for two reasons. The first is that only if the source is offset from the system pointing direction can the one-dimensional radial system acceptance be extracted from the data set under study (because of the exclusion of the source region for acceptance generation). The second reason is that a spectral analysis becomes more difficult and one introduces systematic difficulties for extended sources since the reflected-region method cannot be applied. In fact, for the reflected-region background, $\Theta_{\text {opt }}$ is a compromise between the number of available $O F F$ regions (which increases with increasing offset) and the fall-off of the system acceptance for large offsets. In Fig. 10 we explore the significance as function of wobble offset. For point sources in HESS, $S / \sqrt{t}$ exhibits a rather flat plateau between $0.4^{\circ}$ and $0.7^{\circ}$. For moderately extended sources $\left(\sigma_{\text {source }} \sim 0.2^{\circ}\right)$ the optimal offset increases to $0.7^{\circ}-1.0^{\circ}$. For very large sources the best strategy is to observe just outside of the source, with some safety margin to account for the finite angular resolution, so that one reflected $O F F$ region is available without $\gamma$-ray contamination. Targeted HESS observations are typically taken at $0.5^{\circ}$ or $0.7^{\circ}$ offsets.

\section{Conclusions}

Several different background models are available for ground based Cherenkov astronomy. Different models are appropriate for different purposes. Searches for weak sources are best performed with the robust ring-background model. For spectral 


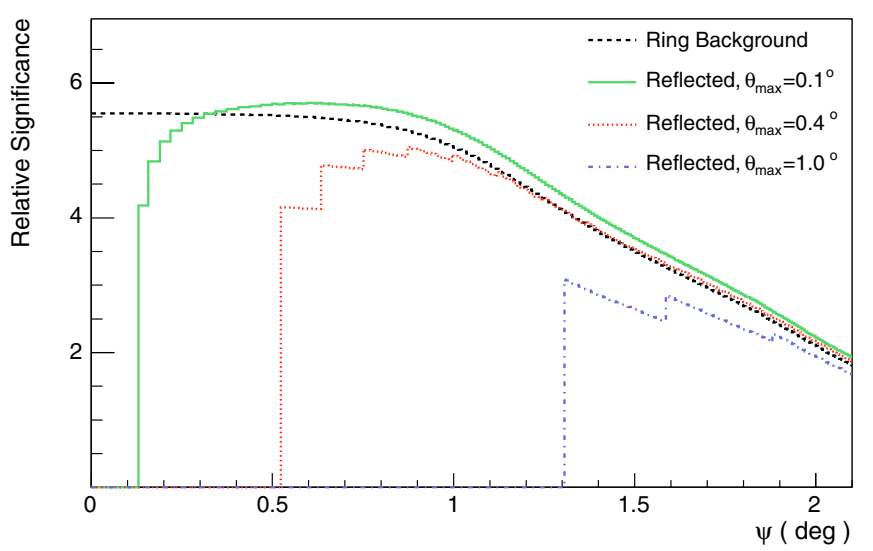

Fig. 10. The impact of an offset between observation position and target source (wobble offset) on the source significance. For a reflected-region background an offset is required to provide $O F F$ regions at equal offset. The relative significance has a broad plateau between 0.5 and 0.7 degrees for point-like sources.

analysis the reflected-region background is favoured. Extracting the morphology of extended sources is often most reliable with the field-of-view or template models. In general, a comparison of several models (with different systematics) is necessary to establish the existence of a new source. It is important to remember that the estimated statistical significance of a source is largely irrelevant if background systematics are not under control.

Acknowledgements. The authors would like to acknowledge the support of their host institutions, and additionally support from the German Ministry for
Education and Research (BMBF). Specifically, JH acknowledges the support of the BMBF through Verbundforschung Astro-Teilchenphysik $(05 \mathrm{CH} 5 \mathrm{VH} 1 / 0)$. We would like to thank the whole HESS collaboration for their support, especially Werner Hofmann, Gerd Pühlhofer, Gavin Rowell and Christian Stegmann for their useful remarks, and Richard White for his careful reading of the manuscript.

\section{References}

Aharonian, et al. (HEGRA Collaboration) 2001, A\&A, 370, 112 Aharonian, et al. (HESS Collaboration) 2004, Nature, 432, 75 Aharonian, et al. (HESS Collaboration) 2005a, A\&A, 439, 1013 Aharonian, et al. (HESS Collaboration) 2005b, Science, 307, 1938 Aharonian, et al. (HESS Collaboration) 2005c, A\&A, 437, L7 Aharonian, et al. (HESS Collaboration) 2005d, A\&A, 430, 865 Aharonian, et al. (HESS Collaboration) 2005e, A\&A, 437, 135 Aharonian, et al. (HESS Collaboration) 2006a, ApJ., 636, 777 Aharonian, et al. (HESS Collaboration) 2006b, A\&A, 449, 223 Aharonian, et al. (HESS Collaboration) 2006c, A\&A, 457, 899 Daum, A., Hermann, G., Hess, M., et al. 1997, Astropart. Phys., 8, 1 Funk, S., Hermann, G., Hinton, J., et al. 2004, Astropart. Phys., 22, 285 Hillas, A. M. 1985, Proc. 19th ICRC, 445

Hinton, J. A. (HESS Collaboration) 2004, New Astron. Rev., 48, 331 Kerrick, A. D., Akerlof, C. W., Biller, S., et al. 1995, ApJ, 452, 588

Kubo, H., Asahara, A., Bicknell, G. V., et al. 2004, New Astron. Rev. 48, 323 Lemoine-Goumard, M., \& Degrange, B. 2005, Proc. Towards a Network of Atmospheric Cherenkov Detectors VII, Palaiseau (France), 173

Li, T., \& Ma, Y. 1983, ApJ, 272, 317

Lorenz, E. (MAGIC Collaboration) 2004, New Astron. Rev., 48, 339 Pühlhofer et al. 2003, Proc. 28th ICRC (Tsukuba), (Tokyo: Univ. Academy Press), 2319 Rowell, G. P. 2003, A\&A, 410, 389

Weekes, T. C., Cawley, M. F., Fegan, D. J., et al., 1989, ApJ, 342, 379

Weekes, T. C., Badran, H., Biller, S. D., et al., 2002, Astropart. Phys., 17, 221 